\title{
Warming up D3 brane motion in the background of D5 brane and inflation
}

\author{
Anindita Bhattacharjee, Atri Deshamukhya ${ }^{\mathrm{a}}$ \\ Department of Physics, Assam University, Silchar, Assam 788011, India
}

Received: 25 March 2014 / Accepted: 16 October 2014 / Published online: 5 November 2014

(c) The Author(s) 2014. This article is published with open access at Springerlink.com

\begin{abstract}
The position of a mobile D-3 brane moving towards a stack of localized D-5 branes has been studied as a candidate driving inflation in the warm-inflationary scenario. Here we consider the dissipation parameter $\Gamma$ as an arbitrary function of only the inflaton field. We find that the observables remain well within PLANCK predictions for a range of model parameters. We also discuss the non-gaussianity generated during inflation in this model.
\end{abstract}

\section{Introduction}

In the past few years there have been many attempts to build cosmological inflation models in the framework of string theory. One of the popular class of models is based on a scenario with a brane moving toward a localized antibrane, where the position of the mobile brane plays the role of the inflaton field [1-19]. In another approach, for inflation model building, the open string tachyon field described by a nonstandard action [20], which lives in a world volume of a nonBPS brane, has been used as the inflaton field [21-28]. It was observed that the same non-standard tachyon effective action also describes the dynamics of a mobile BPS brane in the background of a stack of BPS branes [29-31]. Inflationary models have been constructed in this set-up [32-36]. In particular, an inflation model, where a D3 brane moves in the background of a stack of $k$ coincident D5 branes was investigated in [37]. It was noted that the inflation ends much before the mobile brane comes to a distance of the order of the string length scale, from the stack of localized branes. Thus, the tachyon field, which would have been excited, when they are close enough, does not play any role in driving the inflation.

However, from a thermodynamic viewpoint, there are two dynamical realizations of inflation. The earlier version is the

\footnotetext{
a e-mail: atri.deshamukhya@gmail.com
}

standard inflation scenario (also known as supercooled inflation) where radiation is red-shifted during expansion and leads to a vacuum dominated universe. This gives an isentropic perspective of the inflation paradigm where the universe expands almost exponentially in the inflation phase and as a result its temperature decreases rapidly. Radiation is being introduced through a reheating period after the end of inflation. The fluctuations during this inflation phase are zero-point ground state fluctuations and inflaton field evolution is governed by the ground state evolution equation. There are no thermal perturbations and, therefore, density perturbations here are only adiabatic in nature. In these types of models expansion and reheating are two distinguished phases. Also, energy transfer from potential energy to radiation remains a nontrivial aspect of supercooled inflation [38]. Cold inflation, in fact, is an idealized situation where the dynamics reduces to the classical evolution of the scalar inflaton field with vacuum quantum fluctuations superposed on this background field.

In contrast to the cold-inflationary picture, warm inflation, the other thermodynamic alternative, presents the attractive feature of avoiding the reheating period altogether $[39,40]$. In such type of models dissipative effects are important during the inflationary era, so that radiation production can occur together with the inflationary expansion. The dissipating effect is the result of the friction arising from the scalar field dissipating into a thermal bath via its interaction with other fields during the period of inflation [41]. Phenomenologically in the interaction process, the inflaton decays into some other fields and the decay of the scalar field can be described by means of an interaction Lagrangian. From the point of view of statistical mechanics, the interaction between quantum fields and a thermal bath could be demonstrated by a general fluctuation-dissipation relation [42]. Warm inflation shows how thermal fluctuations may play a dominant role in producing the initial fluctuations necessary for the formation of large-scale structures. Here, 
the density fluctuations arise from thermal rather than quantum fluctuations as happens in supercooled inflation [4346]. These fluctuations have their origin in the hot radiation and their influence on the inflaton scalar field is introduced through a friction term in its equation of motion [47].

Warm inflation was criticized on the basis that the inflaton field cannot decay during the slow-roll phase [48] of inflationary expansion. However, it can be shown that the inflaton field can indeed decay during the slow-roll phase (see [49-51] and references therein), thereby placing the concept of the warm-inflationary paradigm on solid theoretical ground. Over the years, the theory of the dissipation coefficient has met with immense success in the high temperature regime with the condition $T_{\gamma}>m_{\chi}$ where $\chi$ is the field interacting with the inflaton and $T_{\gamma}$ is the radiation temperature. The warm-inflationary scenario in the low temperature regime is also of great interest nowadays which involves a two-stage decay procedure $\phi \rightarrow \chi \rightarrow y y$ where $\chi$ is the heavy intermediate field and $y$ is the finally decaying weak field [52]. As the decay processes in the two regimes are not the same, the density perturbation can be expressed in terms of the dissipation coefficients with a different temperature dependence-which will certainly give rise to different observational consequences [53]. Recently Bastero-Gil et al. have obtained an expression for the associated dissipation coefficient in supersymmetric models [54] in the low temperature regime. Since for cosmological models the space time has to be de Sitter, which does not admit supersymmetry, we refrain from this form of $\Gamma$ in our analysis. In the warm-inflationary scenario, in the presence of radiation in the early universe, the idealization in general made is that of a perfect fluid whereas some deviations might be there from this limit leading to viscous dissipation and corresponding noise forces which might have observational consequences [55].

Warm inflation ends when the universe heats up to become radiation dominated. At this epoch, the universe stops inflating and smoothly enters into a radiation dominated Big Bang phase [56,57]. The matter components of the universe are created by the decay of either the dominant radiation field or the remaining inflationary field [58].

Warm inflation has been studied in the context of tachyon cosmology $[59,60]$, the brane-antibrane scenario $[41,61]$ and the geometric-tachyon driven case [62]. In the present piece of work, we revisit the inflationary scenario driven by the radion field of separation between a D3 and a stack of D5 branes in presence of thermal bath. In Sect. 2 we describe the warm-inflationary scenario in the present context considering the dissipation coefficient an arbitrary function of the inflaton field. Section 3 is dedicated to a discussion and our conclusion.

\section{Warm inflation driven by the radion field between D3 brane and stake of $\mathrm{D} 5$ branes}

\subsection{Review of formalism}

The dynamics of a warm-inflationary model where the inflaton field can be described by a non-standard tachyonic action for a flat FRW metric are described by the equations [59]

$H^{2}=\frac{1}{3 M_{\mathrm{P}}^{2}} \frac{V}{\sqrt{1-\dot{T}^{2}}}$,

$\frac{\ddot{T}}{\sqrt{1-\dot{T}^{2}}}+3 H \dot{T}+\frac{\Gamma \dot{T}}{V} \sqrt{1-\dot{T}^{2}}=-\frac{V_{, T}}{V}$,

and

$\dot{\rho}_{\gamma}+4 H \rho_{\gamma}=\Gamma \dot{T}^{2}$,

where $M_{\mathrm{P}}$ is the reduced Planck mass and $V_{, T} \equiv \partial V / \partial T$; overdots represent derivatives with respect to real time. $\Gamma$ is the dissipation coefficient resulting from the decay of the scalar field into radiation during the inflationary epoch and $\rho_{\gamma}$ is the energy density due to radiation. $\Gamma$ must satisfy $\Gamma>0$ by the second law of thermodynamics. In the analysis of this section, we consider $\Gamma$ as an arbitrary function of only the scalar field, though in principle it should be a function of both scalar field and temperature.

Now to have an inflationary scenario, the necessary conditions are $\rho_{T} \sim V$ and $\rho_{T}>\rho_{\gamma}$. Also, the slow-roll approximation requires that $\dot{T}^{2} \ll 1$ and $\ddot{T} \ll\left(3 H+\frac{\Gamma}{V}\right) \dot{T}$. Under these conditions, the evolution equations can be written as [59]

$H^{2}=\frac{V}{3 M_{\mathrm{P}}^{2}}$

$3 H(1+r) \dot{T}=-\frac{V, T}{V}$,

where we have defined a dimensionless parameter $r$ by $r \equiv \frac{\Gamma}{3 H V}$.

In addition, to have a quasi-stable radiation production during the inflationary epoch, the necessary conditions are $\dot{\rho_{\gamma}} \ll 4 H \rho_{\gamma}$ and $\dot{\rho_{\gamma}} \ll \Gamma \dot{T}^{2}$. With slow-roll conditions, we then have

$\rho_{\gamma}=\frac{\Gamma \dot{T}^{2}}{4 H}$.

On the other hand $\rho_{\gamma}$ can be written as $\rho_{\gamma} \equiv \sigma T_{\gamma}{ }^{4}$ where $\sigma$ is the Stephan-Boltzmann constant and $T_{\gamma}$ is the temperature of the thermal bath.

By using Eqs. 5 and 6 with the definition of $r$, we get

$\rho_{\gamma} \equiv \sigma T_{\gamma}{ }^{4}=\frac{r M_{\mathrm{P}}^{2}}{4(1+r)^{2}}\left(\frac{V, T}{V}\right)^{2}$. 
The combination $\sigma T_{\gamma}{ }^{4}$ can be chosen as a dimensionful parameter $d$ and all the observable quantities in a warm tachyonic inflationary scenario can be expressed in terms of this parameter.

The dimensionless slow-roll parameters in this model are expressible as

$\varepsilon \equiv-\frac{\dot{H}}{H^{2}}=\frac{M_{\mathrm{P}}^{2}}{2(1+r) V}\left(\frac{V, T}{V}\right)^{2}$

$\eta \equiv-\frac{\ddot{H}}{H \dot{H}}=\frac{M_{\mathrm{P}}^{2}}{(1+r) V}\left[\frac{V, T T}{V}-\frac{1}{2}\left(\frac{V,_{T}}{V}\right)^{2}\right]$.

With $r=0$, the above equations reproduce the usual coldinflation expressions. The inflation ends when either of the parameters $\varepsilon$ or $\eta$ goes to one (whichever is early). The number of e-folds from an arbitrary field value to the end of inflation is given by

$N(T)=-\frac{1}{M_{\mathrm{P}}^{2}} \int_{T}^{T_{\mathrm{e}}} \frac{V^{2}}{V, T_{T^{\prime}}}(1+r) \mathrm{d} T^{\prime}$,

where $T_{\mathrm{e}}$ is the field magnitude at the end of inflation. For perturbations, both scalar and tensor, we note that in the case of scalar perturbations the scalar and the radiation fields are interacting. Therefore, isocurvature (or entropy) perturbations are generated besides the adiabatic ones. In this context dissipative effects can produce a variety of spectra, ranging between red and blue, and thus producing the running blue to red spectra [41].

At the high dissipation regime, the density perturbations are expressed as [59]

$\delta_{H}=\frac{16 \pi}{5} \frac{M_{\mathrm{P}}^{2} \exp (-\bar{\zeta}(T)}{(\ln (V))_{T}} \delta T$,

where

$$
\begin{aligned}
\bar{\zeta}(T)= & -\int\left[\frac{1}{3 H r}\left(\frac{\Gamma}{V}\right),_{T}\right. \\
& \left.+\frac{9}{8}\left(1-\frac{(\ln (\Gamma)),_{T}(\ln (V)),_{T}}{36 H^{2} r}\right)(\ln (V)),_{T}\right] \mathrm{d} T \\
= & -\int\left[2 \frac{V, T T}{V,_{T}}-\frac{3 V,_{T}}{8 V}-\frac{3 d V,_{T T}}{4 V V,_{T}}+\frac{3 d V,_{T}}{16 V^{2}}\right] \mathrm{d} T .
\end{aligned}
$$

In terms of the slow-roll parameter $\varepsilon$, the quantity $\delta_{H}^{2}$ can be expressed as

$$
\delta_{H}^{2}=\frac{\sqrt{3}}{75 \pi^{2}} \exp [-2 \bar{\zeta}(T)]\left[\left(\frac{1}{\varepsilon}\right)^{3}\left(\frac{9 M_{\mathrm{P}}^{4}}{2 r^{2} \sigma V}\right)\right]^{\frac{1}{4}},
$$

which gives rise to expressions for various cosmological observables. For example, the spectral index defined by
$n_{\mathrm{S}} \equiv 1+\frac{\mathrm{d} \ln \delta_{H}^{2}}{\mathrm{~d} \ln k}$ becomes

$n_{\mathrm{s}}=1-\left[\frac{3 \eta}{2}+\varepsilon\left[\frac{2 V}{V,_{T}}\left(2 \bar{\zeta}(T)-\frac{r_{T}}{4 r}\right)-\frac{5}{2}\right]\right]$

and the running spectral index is found to be

$$
\begin{aligned}
\alpha_{\mathrm{s}} \equiv & \frac{\mathrm{d} n_{\mathrm{s}}}{\mathrm{d} \ln k} \\
= & -\frac{2 V \varepsilon}{V,_{T}}\left[\frac{2 \eta, T}{2}+\frac{\varepsilon_{T}}{\varepsilon}\left[n_{\mathrm{S}}-1+\frac{3 \eta}{2}\right]\right. \\
& +2 \varepsilon\left[\left(\frac{V}{V, T}\right),_{T}\left(2 \bar{\zeta}_{T}-\frac{(\ln (r)))_{T}}{4}\right)\right. \\
& \left.\left.+\left(\frac{V}{V, T}\right)\left(2 \bar{\zeta}_{T T}-\frac{(\ln (r)),_{T T}}{4}\right)\right]\right] .
\end{aligned}
$$

Similarly, the power spectrum, defined by

$P \equiv \frac{25}{4} \delta_{H}^{2}$,

is expressible as

$P=\frac{1}{4 \pi^{2}}\left[\frac{1}{\sigma d} \frac{V^{6}}{(V, T)^{4}}\right]^{\frac{1}{4}} \exp (-2 \bar{\zeta}(T))$

and the tensor to scalar ratio is given by

$R \equiv\left[\frac{A_{g}^{2}}{P}\right]_{k=k_{0}}$,

where $A_{g}^{2}$ is called the tensor spectrum, and is expressed as

$A_{g}^{2}=\frac{H^{2}}{2 \pi^{2} M_{\mathrm{P}}^{2}}\left[\operatorname{coth}\left[\frac{k}{2 T_{\gamma}}\right]\right]_{k=k_{0}}$.

2.2 Warm inflation driven by the Radion field

When we consider the motion of a D3 brane in the background of $k$ coincident D5 branes, in presence of a radiation bath the scalar field describing the distance between them becomes tachyonic and can be expressed as

$T(R)=\sqrt{L^{2}+R^{2}}+\frac{1}{2} L \ln \frac{\sqrt{L^{2}+R^{2}}-L}{\sqrt{L^{2}+R^{2}+L}}$,

where $R$ is the distance between the moving D3 and $k$ number of static D5 branes. $L$ is defined as $L=\sqrt{k g_{\mathrm{s}} l_{\mathrm{s}}^{2}}, g_{\mathrm{s}}$ being the string coupling and $l_{\mathrm{s}}$ being the string length scale.

Now, for this tachyonic field $T$, the potential function can be written as [37]

$V(T)=\tau_{3} \frac{x}{\sqrt{x^{2}+1}}=\tau_{3} V(x)$, 
where $\tau_{3}$ is the tension on the branes and $x=\frac{R}{L}$ is related to $T$ by

$\Rightarrow \frac{\mathrm{d} T}{\mathrm{~d} x}=\frac{L}{V(x)}$.

Thus, in terms of the dimensionless parameter $x$, the tachyonic field $T$ can be expressed as

$T=L\left(\sqrt{1+x^{2}}+\frac{1}{2} \log \left(\frac{\sqrt{1+x^{2}}-1}{\sqrt{1+x^{2}}+1}\right)\right)$.

In terms of the parameters $r$ and $d$ defined by $r \equiv \frac{\Gamma}{3 H V}=$ $\frac{M_{\mathrm{P}}^{2}\left(V_{, T}\right)^{2}}{4 d V^{2}}$ and $d \equiv \sigma\left(T_{\gamma}\right)^{4}$, the number of e-folds from some initial time to the end of inflation is found to be

$$
\begin{aligned}
N \equiv \int_{t_{i}}^{t_{f}} H \mathrm{~d} t & =-\int_{T}^{T_{f}} \frac{3 H^{2} r V(T)}{V, T} \mathrm{~d} T \\
& =p\left[V(x)-V\left(x_{f}\right)\right] \\
& =p\left[\frac{x}{\sqrt{x^{2}+1}}-\frac{x_{f}}{\sqrt{x_{f}^{2}+1}}\right],
\end{aligned}
$$

where $p$ is another parameter defined as $p \equiv \frac{\tau_{3}}{4 d}$.

Accordingly, the slow-roll parameter $\varepsilon$ and $\eta$ take the forms

$\varepsilon=\frac{2 d}{\tau_{3} V(x)}=\frac{1}{2 p V(x)}$

and

$\eta=\frac{1}{2 p V(x)}\left(1-6 x^{2}\right)=\varepsilon\left(1-6 x^{2}\right)$.

From Eqs. 26 and 27, it is evident that epsilon approaches 1 faster as the field rolls from higher to lower value for any value of the parameter $p$ so long as $x \leqslant 1$,i.e. $R \leqslant L$. Hence the end of inflation is marked by the condition

$\frac{1}{2 p V(x)}=1$,

which in turn gives

$x_{f}=\frac{1}{\sqrt{4 p^{2}-1}}$.

Putting the value of $x_{f}$ in the expression of $N, x$ becomes

$x=\frac{1}{\sqrt{\frac{p^{2}}{\left(N+\frac{1}{2}\right)^{2}}-1}}$.

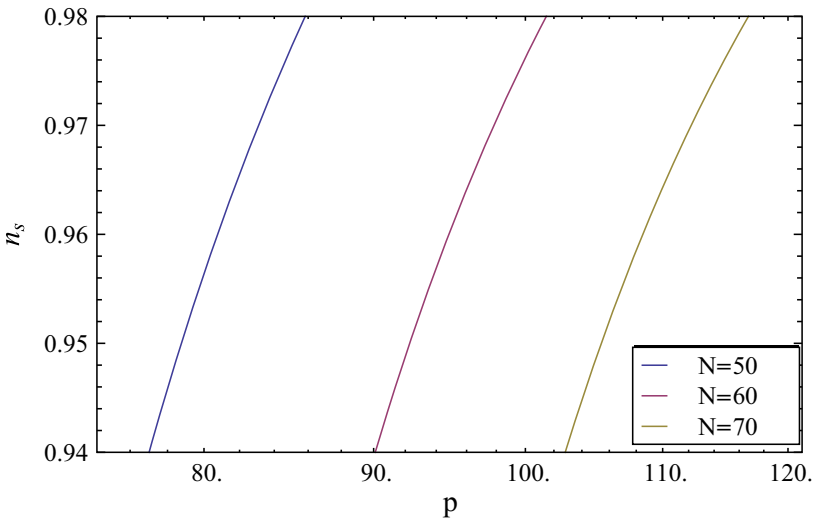

Fig. 1 Variation of the spectral index with $p$ for $N=70$ (brown), 60 (red) and 50 (blue), respectively

Thus, we see that the parameter $x$ can be expressed in terms of $p$ and $N$. It may also be noted that $R \leqslant L$ imposes the constraint $p \geqslant 86$.

After the introduction of all these parameters, we are now ready to compute all the observables which arise from the perturbation spectrum of CMB.

\subsubsection{Perturbational analysis}

\section{(a) Spectral index}

The spectral index in terms of the model parameter $p$ may be expressed as

$$
\begin{aligned}
n_{\mathrm{s}}= & 1-\left[\frac{3 \eta}{2}+\varepsilon\left[\frac{2 V}{V, T}\left(2 \bar{\zeta}(T)-\frac{r_{T}}{4 r}\right)-\frac{5}{2}\right]\right] \\
= & \frac{3 d^{2}}{2 V^{2}}+\frac{3 d}{V}+1-\frac{6 d^{2} V, T T}{V(V, T)^{2}}+\frac{12 V,,_{T T} d}{(V, T)^{2}} \\
= & 1+\left(\frac{3}{32 p^{2} V(x)}+\frac{3}{4 p}\right) \frac{1}{V(x)}-\frac{3}{8 p^{2}} \\
& \times\left(\frac{1}{V(x)^{2}}+\frac{V^{\prime \prime}(x)}{V(x) V^{\prime}(x)^{2}}\right)+\frac{3}{p}\left(\frac{1}{V(x)}+\frac{V^{\prime \prime}(x)}{V^{\prime}(x)^{2}}\right) \\
= & 1+\frac{3\left[-3+x\left(40 p \sqrt{1+x^{2}}+3 x\left[3+4 x\left(x-8 p \sqrt{1+x^{2}}\right)\right]\right)\right]}{32 p^{2} x^{2}} .
\end{aligned}
$$

As $x$ is expressible as a function of both $p$ and $N$, the spectral index can be expressed in terms of the parameter $p$ and the number of e-foldings $N$ before the end of inflation at which the observables are evaluated.

In Fig. 1, the variation of the spectral index with respect to $p$ for three different $N$ values are shown. It can be seen that for a wide range of the parameter $p$, the value of $n_{\mathrm{s}}$ lies well within the limit viz. $n_{\mathrm{s}}=0.09603 \pm 0.0073$ predicted by PLANCK [63]. A reanalysis, however, yields $n_{\mathrm{S}}=0.9608 \pm 0.0057$ (68\% C.L.) from Planck + WP, BAO and Union2.1 compilation data [64]. It may be worth noting that the inclusion of an effective number of neutrino 
species could significantly weaken the constraint, namely $n_{\mathrm{s}}=0.9693 \pm 0.0079$ (68\% C.L.).

\section{(b) Running of spectral index}

The running of the spectral index is another observable associated with the spectral index, and it can be defined as

$$
\begin{aligned}
& \alpha_{\mathrm{s}}=-\frac{2 V \varepsilon}{V,_{T}}\left[\frac{2 \eta, T}{2}+\frac{\varepsilon_{T}}{\varepsilon}\left[n_{\mathrm{S}}-1+\frac{3 \eta}{2}\right]\right. \\
& +2 \varepsilon\left[\left(\frac{V}{V, T}\right), T\left(2 \bar{\zeta}, T-\frac{(\ln (r)),_{T}}{4}\right)\right. \\
& \left.\left.+\left(\frac{V}{V, T}\right)\left(2 \bar{\zeta}, T T-\frac{(\ln (r))_{T T}}{4}\right)\right]\right] \\
& =-\frac{12 d^{2}}{V^{3}\left(V,_{T}\right)^{4}}\left[(d+V)\left(V,_{T}\right)^{4}-2 d V\left(V,_{T}\right)^{2} V,_{T T}\right. \\
& \left.+4 V^{2}[-d+2 V]\left(V,{ }_{T T}\right)^{2}+2[d-2 V] V^{2} V,_{T} V,_{T T T}\right] \\
& =-\left(\frac{3}{16 p^{3} V(x)^{3}}+\frac{3}{4 p^{2} V(x)^{2}}\right. \\
& -\frac{3\left[V(x) V^{\prime}(x)^{2}+V(x)^{2} V^{\prime \prime}(x)\right]}{8 p^{3} V(x)^{4} V^{\prime}(x)^{2}} \\
& -\frac{3\left[V(x) V^{\prime}(x)^{2}+V(x)^{2} V^{\prime \prime}(x)\right]^{2}}{4 p^{3} V(x)^{5} V^{\prime}(x)^{2}} \\
& +\frac{6\left[V(x) V^{\prime}(x)^{2}+V(x)^{2} V^{\prime \prime}(x)\right]^{2}}{p^{2} V(x)^{4} V^{\prime}(x)^{2}} \\
& +\frac{3\left[V(x) V^{\prime}(x)^{3}+4 V(x)^{2} V^{\prime}(x) V^{\prime \prime}(x)+V(x)^{3} V^{\prime \prime \prime}(x)\right]}{8 p^{3} V(x)^{4} V^{\prime}(x)^{4}}
\end{aligned}
$$

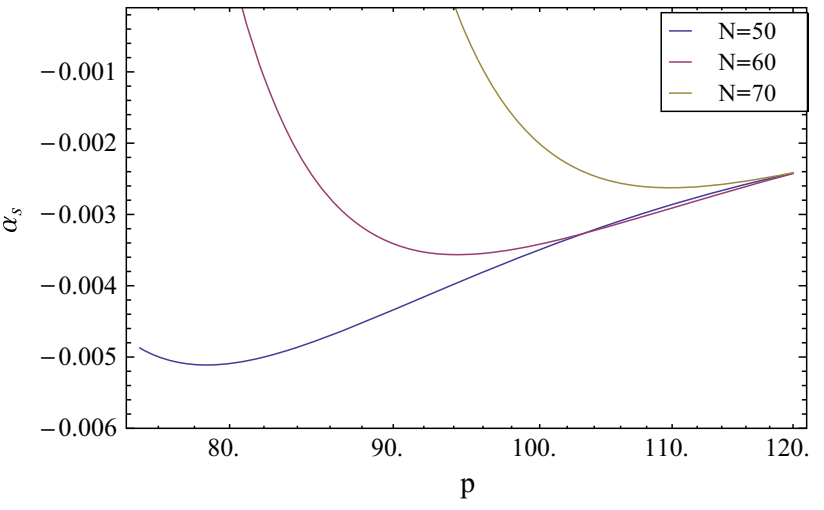

Fig. 2 Variation of the running of the spectral index with respect to $p$ for $N=70$ (brown), 60 (red) and 50 (blue), respectively

$$
\begin{aligned}
& +x^{2}\left(-20+27 \sqrt{1+x^{2}}+x^{2}\left(-106-21 \sqrt{1+x^{2}}\right.\right. \\
& +x^{2}\left(-160+92 x^{4}+90 x^{6}+24 x^{8}+17 \sqrt{1+x^{2}}\right. \\
& \left.\left.\left.\left.+x^{2}\left(-50+6 \sqrt{1+x^{2}}\right)\right)\right)\right)\right] .
\end{aligned}
$$

In Fig. 2, the running of the spectral index is plotted against the parameter $p$ and it is found that in the range of parameter $p$ for which the $\alpha_{\mathrm{s}}$ is within the limit predicted by PLANCK [63], viz. $\alpha=-0.013 \pm 0.009$, we have a subset of the parameter range obtained for $n_{\mathrm{s}}$.

\section{(c) Power spectrum}

The power spectrum in terms of the model parameters can be expressed as

$$
\begin{aligned}
& P=\frac{1}{4 \pi^{2}}\left[\frac{1}{\sigma d} \frac{V^{6}}{(V, T)^{4}}\right]^{\frac{1}{4}} \exp (-2 \bar{\zeta}(T)) \\
& =\frac{\exp \left[\frac{-3 \log [V(x)]}{8 p}-\frac{3 \log \left[V^{\prime}(x)\right]}{8 p}-\frac{3}{32 p V(x)}\right] V(x)^{\frac{13}{4}} V^{\prime}(x)^{4}\left[\frac{f p^{2} V(x)^{2}}{V^{\prime}(x)^{4}}\right]^{\frac{1}{4}}}{4 \pi^{2}} \\
& =\frac{1}{4 \pi^{2}} \exp \left[\frac{-3 \sqrt{1+x^{2}}}{32 p x}-\frac{3 \log \left(\frac{x}{\sqrt{x^{2}+1}}\right)}{8 p}-\frac{3 \log \left(\frac{-x^{2}}{\left(1+x^{2}\right)^{\frac{3}{2}}}+\frac{1}{\sqrt{x^{2}+1}}\right)}{8 p}\right]\left[\frac{x}{\sqrt{x^{2}+1}}\right]^{\left(\frac{13}{4}\right)}\left[\frac{f p^{2} x^{2}}{\left(1+x^{2}\right)\left(\frac{-x^{2}}{\left(1+x^{2}\right)^{\frac{3}{2}}}+\frac{1}{\sqrt{x^{2}+1}}\right)^{4}}\right]^{\frac{1}{4}} \\
& \times\left[\frac{1}{\sqrt{x^{2}+1}}-\frac{x^{2}}{\left(1+x^{2}\right)^{\frac{3}{2}}}\right]^{4} \\
& \left.-\frac{3\left[V(x) V^{\prime}(x)^{3}+4 V(x)^{2} V^{\prime}(x) V^{\prime \prime}(x)+V(x)^{3} V^{\prime \prime \prime}(x)\right]}{p^{2} V(x)^{3} V^{\prime}(x)^{3}}\right) \\
& =-\frac{3}{16 p^{3} x^{3}\left(1+x^{2}\right)^{2}}\left[2-5 \sqrt{1+x^{2}}\right. \\
& +4 p x\left(5+3 x^{2}+195 x^{4}+33 x^{5}-84 x^{8}-48 x^{10}\right)
\end{aligned}
$$

where we have defined a dimensionless parameter $f$ as $f \equiv$ $\frac{16 L^{4}}{\sigma}$. The value of the parameter $f$ can be found from the observational constraint on the power spectrum as predicted by $\mathrm{COBE}$ normalization condition i.e. $P=2 \times 10^{-9}$. The parameter $f$ can be expressed in terms of $p$ and $N$ and hence it is not a new parameter in our model. 


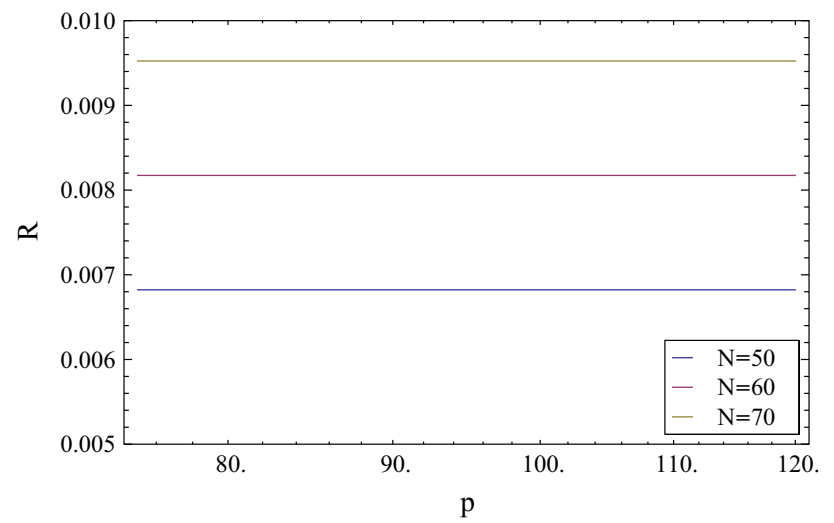

Fig. 3 Variation of $R$ with the parameter $p$ for $N=70$ (brown), 60 (red) and 50 (blue), respectively

\section{(d) Tensor to scalar ratio}

Another observable, the ratio of tensor spectrum to the scalar one, in terms of the model parameters reads values of $N$ and it is found that for each value of $N$, the value of $R$ lies well within the bound predicted by PLANCK i.e. $r<0.11$. But the PLANCK observation is under tension from a recent BICEP2 result [65], which claims the detection of a tensor to scalar ratio $R=0.2_{-0.05}^{+0.07}$. However, with consideration of foreground dust the value has been claimed to be in need to be lowered to $R=0.16_{-0.05}^{+0.06}$ [66]. Also it may be noted that depending upon the modeling of foreground dust the value of $R$ could be lowered to the level of becoming undetectable in observations [67].

\subsubsection{Non-gaussianity}

Non-gaussian statistics provides a powerful way to observationally discriminate between different mechanisms of generating a curvature perturbation. Since a warm-inflationary scenario can be viewed as a multifield inflationary scenario, it may produce large non-gaussianities. In order to study these non-gaussian effects, we need to obtain the three point corre-

$$
\begin{aligned}
R & =\frac{2}{3 M_{\mathrm{P}}^{4}}\left[\frac{\sigma d[V, T]^{4}}{V^{2}}\right]^{\frac{1}{4}} \exp (2 \bar{\zeta}(T))\left[\operatorname{coth}\left[\frac{k}{2 T_{\gamma}}\right]\right]_{k=k_{0}} \\
& =\frac{32 d\left[\frac{p^{2} V(x)^{2} V^{\prime}(x)^{4}}{f}\right]^{\frac{1}{4}} \exp \left[\frac{3 \log [V(x)]}{8 p}+\frac{3 \log \left[V^{\prime}(x)\right]}{8 p}+\frac{3}{32 p V(x)}\right]}{3 V(x)^{\frac{13}{4}} V^{\prime}(x)^{4}} \\
& =\frac{32 d \exp \left[\frac{3 \sqrt{1+x^{2}}}{32 p x}+\frac{3 \log \left(\frac{x}{\sqrt{x^{2}+1}}\right)}{8 p}+\frac{\left.3 \log \left(\frac{-x^{2}}{\left(1+x^{2}\right)^{\frac{3}{2}}}+\frac{1}{8 p}\right)\right]\left[\frac{p^{2} x^{2}\left[\frac{1}{\sqrt{x^{2}+1}}-\frac{x^{2}}{\left(1+x^{2}\right)^{\frac{3}{2}}}\right.}{x^{2}+1}\right]^{4}}{f\left(x^{2}+1\right)}\right]^{\frac{1}{4}}}{3\left[\frac{1}{\sqrt{x^{2}+1}}-\frac{x^{2}}{\left(1+x^{2}\right)^{\frac{3}{2}}}\right]^{4}\left(\frac{x}{\sqrt{x^{2}+1}}\right)} .
\end{aligned}
$$

$R$ can be evaluated by using the observational value of $d$, which is a combination of the radiation temperature $T_{\gamma}$, Stefan's constant $\sigma$ and $k_{0}$. From observation, using the fact that $T_{\gamma} \cong 0.24 \times 10^{16} \mathrm{GeV}, \sigma=1$ and $k_{0}=0.002 M p c^{-1}$ [59], $R$ can be measured in terms of the parameters $p$ and $N$. In Fig. 3, $R$ is plotted against $p$ for three different lation function of the density perturbation or the bispectrum [68]. The bispectrum is expressed in terms of the $f_{N L}$ parameter, the value of which predicts whether non-gaussianity is arising from the inflationary model or not.

In a warm-inflationary scenario, the $f_{N L}$ parameter can be expressed in terms of the potential $V(x)$ as [69]

$$
\begin{aligned}
f_{N L} & =-\frac{5}{3}\left(\frac{\dot{T}}{H}\right)\left(\frac{1}{H} \log \left(\frac{k_{f}}{H}\right)\right)\left[\frac{V, T T T}{\Gamma}+\frac{\left(2 k_{f}\right)^{2} V, T}{\Gamma}\right] \\
& =\frac{5 \log \left[\frac{\sqrt{3} V^{\prime}(x)}{2 \sqrt{d}}\right]\left[\frac{\left[V(x) V^{\prime}(x)^{3}+4 V(x)^{2} V^{\prime}(x) V^{\prime \prime}(x)+V(x)^{3} V^{\prime \prime \prime}(x)\right]}{V(x)^{\frac{3}{2}} V^{\prime}(x)^{2}}+2 p \sqrt{V(x)} V^{\prime}(x)\right]}{3 p^{2} V^{\prime}(x) V(x)^{\frac{3}{2}}} .
\end{aligned}
$$




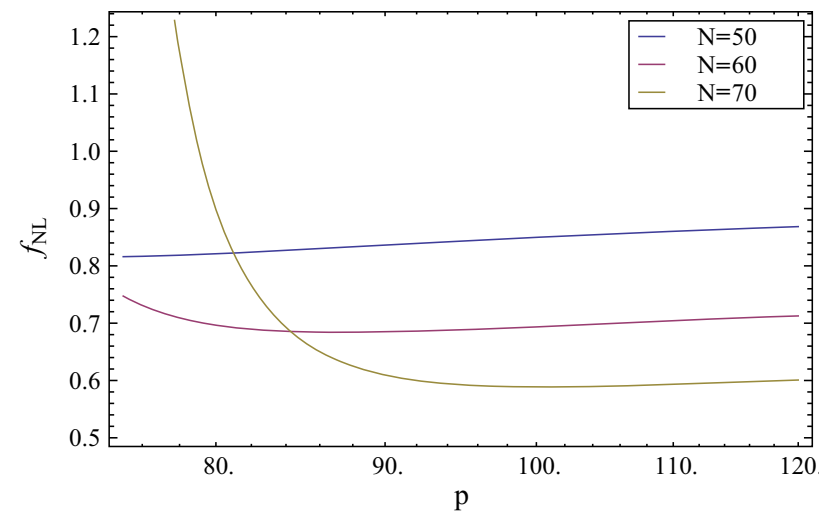

Fig. 4 Variation of $f_{N L}$ with the parameter $p$ for $N=70$ (brown), 60 (red) and 50 (blue), respectively

In this case, $V(x)=\frac{x}{\sqrt{x^{2}+1}}$ and hence in terms of the model parameters

$$
f_{N L}^{f_{N L}}=\frac{5 \log \left[\frac{\sqrt{3}}{2 \sqrt{d}\left(1+x^{2}\right)^{\frac{3}{2}}}\right]\left[1-14 x^{2}-3 x^{4}+12 x^{6}+2 p x \sqrt{x^{2}+1}\right]}{3 p^{2} x^{2}} .
$$

Like the other observables, $f_{N L}$ can also be expressed in terms of the parameter $p$ and $N$.

In Fig. 4, the $f_{N L}$ parameter is plotted against the parameter $p$ for $N=50,60,70$, respectively. It can be seen that the value of this parameter is well within the bound predicted by recent observations of PLANCK [70] for a large range of $p$.

\subsubsection{Constraint on number of D5 branes}

A constraint on the number of D5 branes to realize this model of inflation can be obtained as follows. From the whole analysis, we can see that all the observables lie well within the limit as predicted by recent observation for $p \geqslant 86$ Now at the end point of inflation marked by $\varepsilon=1$, we have

$$
\begin{aligned}
& \frac{1}{2 V(x)}=1 \\
& \Rightarrow \frac{1}{2 p \frac{x}{\sqrt{x^{2}+1}}}=1 \\
& \Rightarrow x=\frac{1}{\sqrt{4 p^{2}-1}} .
\end{aligned}
$$

As $x=\frac{R}{L}$ and $L=\sqrt{k g_{\mathrm{s}} l_{\mathrm{s}}^{2}}$, we have

$$
g_{\mathrm{s}}=\frac{\left(4 p^{2}-1\right) R^{2}}{k l_{\mathrm{s}}^{2}}
$$

Now, using the conditions $R<\sqrt{k g_{\mathrm{s}} l_{\mathrm{s}}^{2}}$ and $g_{\mathrm{s}}<<1$, we arrive at the expression

$k>\left(R / l_{\mathrm{s}}\right)^{2}\left(4 p^{2}-1\right)$.

Now, using $p=100$, the number of branes required is of the order of $k>10^{4}$ and as we increase $p$, this requirement also increases. It may be interesting to note here that for a similar inflationary picture in the absence of a radiation bath [37], the constraint on the number of branes was obtained as $k g_{\mathrm{s}}\left(l_{\mathrm{s}} M_{\mathrm{P}}\right)^{2} \approx 10^{10}$, which roughly leads to the impression that $k \geqslant 10^{10}$.

\section{Conclusion}

In this work, the warm-inflationary scenario driven by a BPS D3 brane moving in the background of $k$ coincident D5 branes has been analyzed. The unstable tachyonic field which arises from the motion of a D3 brane in that background is the source of inflation in this picture. The whole process is considered in the presence of a radiation bath. In such warm inflation, the dissipative effects play an important role and hence the whole dynamics is analyzed in terms of the dissipative parameter. The slow-roll parameters and the cosmological observables are computed by considering the fact that the dissipation parameter is a function of the tachyonic field. The number of e-foldings $N$, the spectral index $n_{\mathrm{s}}$, running of spectral index $\alpha_{\mathrm{s}}$, and the tensor-scalar ratio $R$ are evaluated for the general tachyonic potential in terms of our model parameters. We have carried out the analysis for the three cases $N=50, N=60$, and $N=70$ separately and found that, for each case, all the observables lie well within the bound predicted by the observation of PLANCK for a wide range of the model parameter $p$, though the value of the tensor to scalar ratio is far less than the predictions of BICEP2. The model predicts that by a suitable choice of the parameter $p$, one can get a constraint on the minimum number of $\mathrm{D} 5$ branes required to realize such a model. It is observed that for $p=100$, we need a minimum of $10^{4}$ branes to drive inflation. Also, it is seen that non-gaussian effects can arise in this model due to the self-interaction of the inflaton field. The non-gaussian effect of such inflationary mechanism is analyzed by measuring the bispectrum of the gravitational field fluctuations generated during the warm inflation in the strong dissipative regime. The bispectrum of the inflaton is expressed in terms of the parameter $f_{N L}$ and it can be seen that the value of $f_{N L}$ parameter lies well within observed limits for a wide range of the model parameters.

Acknowledgments Financial support from DAE Project grant 2009/ 24/35 (BRNS) is acknowledged. Hospitality provided by HRI, Allahabad and IUCAA, Pune during preparation of the manuscript is highly 
appreciated. We thank Prof. A. Berera and Prof. S. Panda for valuable discussions.

Open Access This article is distributed under the terms of the Creative Commons Attribution License which permits any use, distribution, and reproduction in any medium, provided the original author(s) and the source are credited.

Funded by $\mathrm{SCOAP}^{3}$ / License Version CC BY 4.0.

\section{References}

1. G.R. Dvali, S.H.H. Tye, Phys. Lett. B 450, 72 (1999)

2. G. R. Dvali, Q. Shafi, S. Solganik, arXiv:hep-th/0105203

3. C.P. Burgess, M. Majumdar, D. Nolte, F. Quevedo, G. Rajesh, R.J. Zhang, JHEP 0107, 047 (2001)

4. C.P. Burgess, P. Martineau, F. Quevedo, G. Rajesh, R.J. Zhang, JHEP 0203, 052 (2002)

5. D. Choudhury, D. Ghoshal, D.P. Jatkar, S. Panda, JCAP 0307, 009 (2003)

6. J. Garcia-Bellido, R. Rabadan, F. Zamora, JHEP 0201, 036 (2002)

7. N. Jones, H. Stoica, S.H.H. Tye, JHEP 0207, 051 (2002)

8. M. Gomez-Reino, I. Zavala, JHEP 0209, 020 (2002)

9. C. Herdeiro, S. Hirano, R. Kallosh, JHEP 0112, 027 (2001)

10. S. Kachru, R. Kallosh, A. Linde, J. Maldacena, L. McAllister, S.P. Trivedi, JCAP 0310, 013 (2003)

11. D. Baumann, A. Dymarsky, I.R. Klebanov, L. McAllister, P.J. Steinhardt, Phys. Rev. Lett. 99, 141601 (2007)

12. A. Krause, E. Pajer, JCAP 0807, 023 (2008)

13. S. Panda, M. Sami, S. Tsujikawa, Phys. Rev. D 76, 103512 (2007)

14. D. Baumann, A. Dymarsky, S. Kachru, I.R. Klebanov, L. McAllister, JHEP 0903, 093 (2009)

15. A. Ali, R. Chingangbam, S. Panda, M. Sami, Phys. Lett. B 674, 131 (2009)

16. S. Panda, M. Sami, I. Thongkool, Phys. Rev. D 81, 103506 (2010)

17. D. Baumann, A. Dymarsky, S. Kachru, I.R. Klebanov, L. McAllister, Phys. Rev. Lett. 104, 251602 (2010)

18. D. Baumann, A. Dymarsky, S. Kachru, I.R. Klebanov, L. McAllister, JHEP 1006, 072 (2010)

19. A. Ali, A Deshamukhya, S. Panda, M. Sami, Eur. Phys. J. C 71, $1672(2011)$

20. E.A. Bergshoeff, M. de Roo, T.C. de Wit, E. Eyras, S. Panda, JHEP 0005, 009 (2000)

21. A. Mazumdar, S. Panda, A. Perez-Lorenzana, Nucl. Phys. B 614, $101(2001)$

22. M. Fairbairn, M.H.G. Tytgat, Phys. Lett. B 546, 1 (2002)

23. G.W. Gibbons, Phys. Lett. B 537, 1 (2002)

24. M. Sami, P. Chingangbam, T. Qureshi, Phys. Rev. D 66, 043530 (2002)

25. Y.S. Piao, R.G. Cai, X. M. Zhang, Y. Z. Zhang. Phys. Rev. D 66, $121301(2002)$

26. D. Choudhury, D. Ghoshal, D.P. Jatkar, S. Panda, Phys. Lett. B 544, 231 (2002)

27. J. Raeymaekers, JHEP 0410, 057 (2004)

28. P. Chingangbam, S. Panda, A. Deshamukhya, JHEP 0502, 052 (2005)
29. D. Kutasov, arXiv:hep-th/0405058

30. D. Kutasov, arXiv:hep-th/0408073

31. A. Das, S. Panda, S. Roy, Phys. Rev. D 78, 061901 (2008)

32. S. Thomas, J. Ward, Phys. Rev. D 72, 083519 (2005)

33. S. Panda, M. Sami, S. Tsujikawa, Phys. Rev. D 73, 023515 (2006)

34. B. Gumjudpai, T. Naskar, J. Ward, JCAP 0611, 006 (2006)

35. E. Papantonopoulos, I. Pappa, V. Zamarias, JHEP 0605, 038 (2006)

36. A. Sen, JHEP 0705, 035 (2007)

37. S. Panda, M. Sami, S. Tsujikawa, J. Ward, Phys. Rev. D 73, 083512 (2006)

38. K. Nojari, M. Shoukrani, Astrophys. Space Sci. 339, 111 (2012)

39. A. Berera, Phys. Rev. Lett. 75, 3218 (1995)

40. A. Berera, Phys. Rev. D 55, 3346 (1997)

41. S. del Campo, R. Herrera, Phys. Lett. B 653, 122-128 (2007)

42. U. Weiss, Quantum Dissipative Systems (World Scientific, Singapore, 1993)

43. L.M.H. Hall, I.G. Moss, A. Berera, Phys. Rev. D 69, 083525 (2004)

44. I.G. Moss, Phys. Lett. B 154, 120 (1985)

45. A. Berera, L.Z. Fang, Phys. Rev. Lett. 74, 1912 (1995)

46. A. Berera, Nucl. Phys. B 585, 666 (2000)

47. A. Berera, Phys. Rev. D 54, 2519 (1996)

48. J. Yokoyama, A. Linde, Phys. Rev. D 60, 083509 (1999)

49. A. Berera, R. O. Ramos, Phys. Rev. D 71, 023513 (2005)

50. L.M. Hall, I.G. Moss, Phys. Rev. D 71, 023514 (2005)

51. M. Bastero-Gil, A. Berera, Phys. Rev. D 71, 063515 (2005)

52. I.G. Moss, Phys. Lett. B 154, 120 (1995)

53. L. M. H. Hall, I. G. Moss, A. Berera, Phys. Rev. D 69, 083525 (2004). astro-ph/0507124

54. M. B. Gil, A. Berera, R. O. Ramos, J. G. Rosa, JCAP 1301, 016 (2013)

55. M. B. Gil, A. Berera, I. G. Moss, R. O. Ramos, arXiv:1401.1149 [astro-ph.CO]

56. A. Berera, Phys. Rev. D 55, 3346 (1997)

57. J. Mimoso, A. Nunes, D. Pavon, Phys. Rev. D 73, 023502 (2006)

58. A.N. Taylor, A. Berera, Phys. Rev. D 62, 083517 (2000)

59. R. Herrera, S. del Campo, C. Campuzano, JCAP 10, 9 (2006)

60. A. Deshamukhya, S. Panda, Int. J. Mod. Phys. D 18, 2093-2106 (2009)

61. M. Bastero-Gil, A. Berera, J.G. Rosa, Phys. Rev. D84, 103503 (2011)

62. A. Bhattacharjee, A. Deshamukhya, Mod. Phys. Lett. A 28, 1350036 (2013)

63. P. Ade et al., PLANCK Collaboration (2013). arXiv:1303.5082

64. H. Li, J. Xia, X. Zhang, Phys. Dark Univ. 2, 188 (2013)

65. P. Ade et al., BICEP2 Collaboration (2014). arXiv:1403.3985

66. M. J. Mortnson, U. Seljak (2014). arXiv:1405.5857 [astro-ph.CO]

67. J. Martin, C. Ringeval, R. Trotta, V. Vennin, arXiv:1405.7272 [astro-ph.CO]

68. S. Gupta, A. Berera, A. F. Heavens, S. Matarrese, Phys. Rev. D 66, 043510 (2002). arXiv:astro-ph/0205125V2

69. M. R. Setare, V. Kamali, JCAP 08, 034 (2012). arXiv:1204.2156 [hep-th]

70. P. Ade et al., Planck Collaboration (2013). arXiv:1303.5084 [astroph.CO] 